LA W REN CE LIVERM ORE NATIONAL LABORATORY

X-Ray Array Sources

S. K. Lehman, A. M. Foudray

October 10, 2011 
This document was prepared as an account of work sponsored by an agency of the United States government. Neither the United States government nor Lawrence Livermore National Security, LLC, nor any of their employees makes any warranty, expressed or implied, or assumes any legal liability or responsibility for the accuracy, completeness, or usefulness of any information, apparatus, product, or process disclosed, or represents that its use would not infringe privately owned rights. Reference herein to any specific commercial product, process, or service by trade name, trademark, manufacturer, or otherwise does not necessarily constitute or imply its endorsement, recommendation, or favoring by the United States government or Lawrence Livermore National Security, LLC. The views and opinions of authors expressed herein do not necessarily state or reflect those of the United States government or Lawrence Livermore National Security, LLC, and shall not be used for advertising or product endorsement purposes.

This work performed under the auspices of the U.S. Department of Energy by Lawrence Livermore National Laboratory under Contract DE-AC52-07NA27344. 


\section{X-Ray Array Sources}

Sean K. Lehman

Angela M. Foudray

The intent of this project was to study the application of x-ray array sources to problems of importance to LLNL. Arrays of acoustic and electromagnetic transducers have existed for decades but the technology to manufacture and operate x-ray array sources have only recently been achieved. In theory, x-ray array sources could reduce data collection times, enhance resolution, increase contrast, and reduce imaging artifacts.

To demonstrate these imaging improvements, LLNL designed the Contrast and Resolution Interleaved Stacked Plate (CRISP) phantom (ref. 1), a stacked disk assembly in which the disk materials alternate in type and attenuation. They may also contain resolution patterns machined into the disks. The goal is to form a contrast or, contrast and resolution phantom. The materials used in the CRISP phantom are Kynar and LDPE. Selected disks contain resolution patterns in the form of holes of varying sizes. Within the stack, selected disks are offset so as to model "As Built" versus "As Designed" issues as well. The assembled phantom is shown in Figure 1.

To achieve its goal, the project engaged Triple Ring Technologies (http://www.tripleringtech.com/), an x-ray array source manufacturer, to collect data on the CRISP phantom. As a comparison, data were collected on a technologically mature single source cone-beam system at LLNL. Whereas cone-beam reconstruction codes are mature, array source reconstruction codes are nascent. LLNL implemented a variation on an expectation maximization (EM) algorithm to reconstruct these data.

Expectation maximization (EM) is an iterative algorithm that computes maximum likelihood estimates for any statistical measurement scheme. In x-ray reconstructions, the statistical nature of the system arises from the stochastic nature of x-ray photon emission. EM also incorporates non-negativity constraints (physically, the number of photons never goes below zero) and measures of reconstructed image quality. Convergence towards a solution (an image) is achieved by iteratively going back and forth between an estimation step, and maximization step. Unknown parameters, such as number of photons emitted by the sources, are estimated in the expectation step; and an image-likelihood is maximized in the maximization step.

Although reliable and robust, EM can be slow for large data sets. A speed up was introduced where the expectation and maximization steps were performed after adding only a subset of the data at a time, instead of the full data set at each update. This new method, ordered subset expectation maximization, (OSEM) is a standard tool for countstarved tomographic imaging modalities, such as the Triple Ring Technologies measurement system. In x-ray measurement systems, data are taken in a physically ordered fashion: a detector head is swept around the object of interest, collecting data in neighboring angular swaths. 
Using data ordered in this way creates artifacts (intensity anisotropies and feature blur). The project developed a randomized subset expectation maximization (RSEM) implementation of the EM algorithm which essentially eliminates these artifacts. The randomized subset implementation can either be run in a mode where subsets are constructed once of angularly randomized data and the same subsets are used in a roundrobin manner to update the EM algorithm. The other method is to create subsets each iteration (each time the entire data set is fully utilized) that are randomized.

Both methods have no artifacts due to event ordering and have been shown to be essentially interchangeable.

As a proof-of-principle the randomized subset expectation maximization (RSEM) offered an improvement over current reconstruction algorithms, a reconstruction was performed of a data set consisting of a steel bar and a container of jelly. The importance of these data lie in the large attenuation contrast between the two materials. Figure 2 presents a comparison of a standard computed tomography (CT) reconstruction algorithm with a modified constrained conjugate gradient (CCG) algorithm and RSEM. The standard algorithm shows increased artifacts and poorer contrast than the other two. The RSEM algorithm yields the best contrast and resolution.

With this, single source, success of the RSEM algorithm, it was used to reconstruct the Triple Ring Technologies array source CRISP phantom data. As a comparison, single source cone beam data were collected on the CRISP phantom and reconstructed. Slices through the reconstructions are presented in Figure 3. Unfortunately, the array source data proved to lack the photon count to achieve a fair reconstruction comparison with the single source data. However, off-angle blurring artifacts appear less in the array source reconstruction. This provides hope that as photon counts and energy levels are increased in array sources, they will out-perform the current single source systems. 


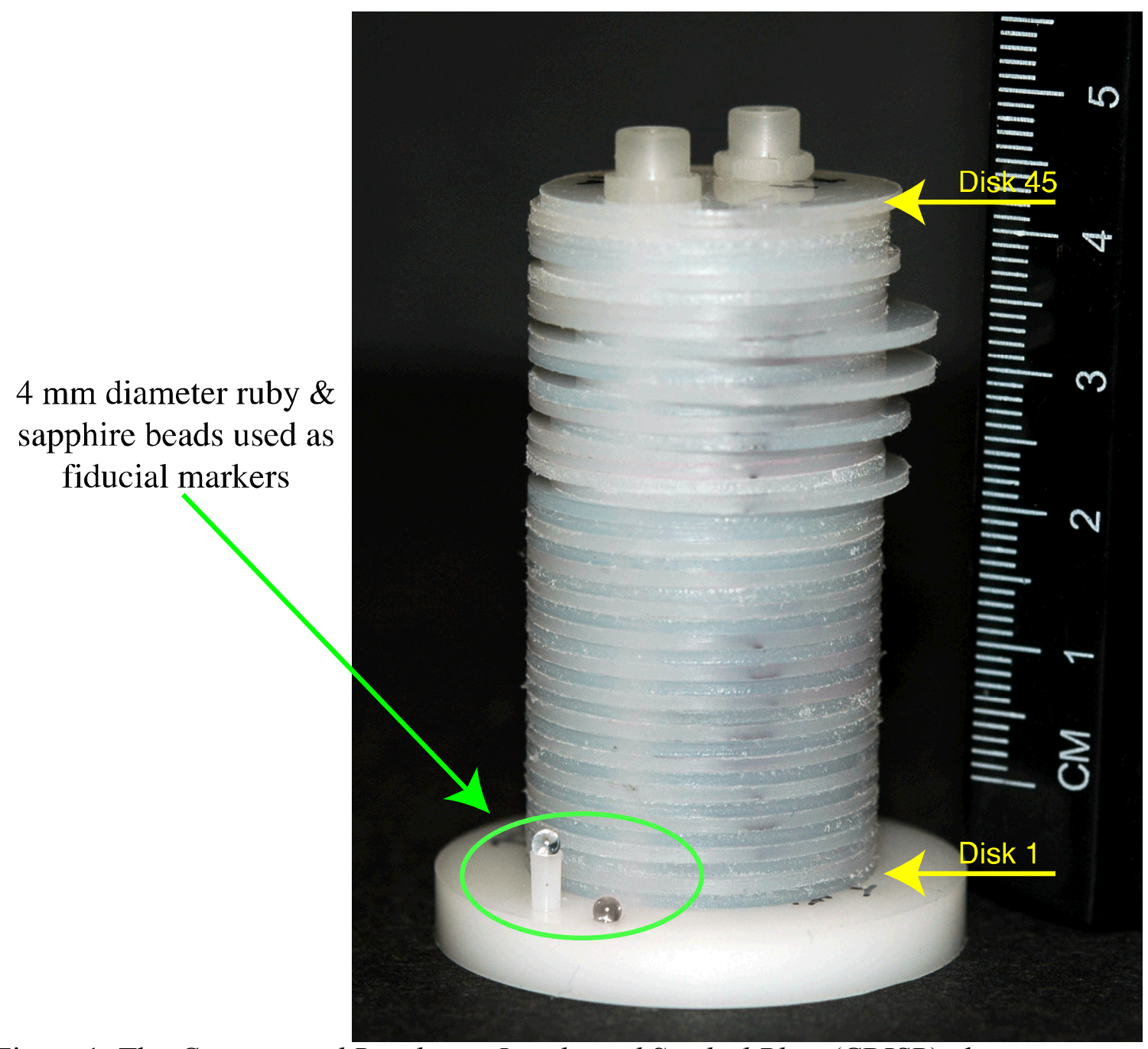

Figure 1: The Contrast and Resolution Interleaved Stacked Plate (CRISP) phantom. 

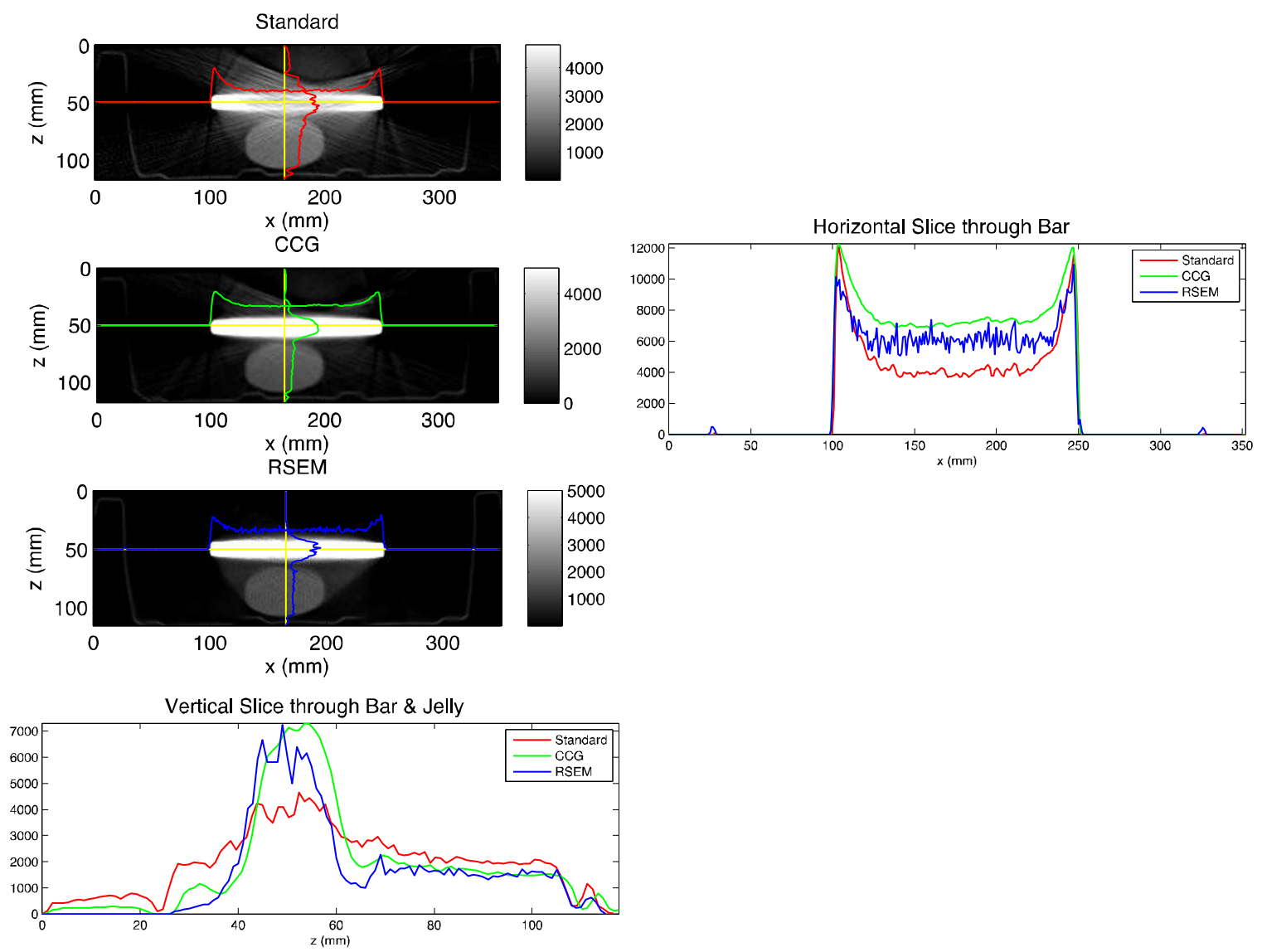

Figure 2: Reconstruction comparisons of a data set consisting of a steel bar and a container of jelly. The algorithms are a standard computed tomography (CT) algorithm, a modified constrained conjugate gradient (CCG) algorithm, and the randomized subset expectation maximization (RSEM). 

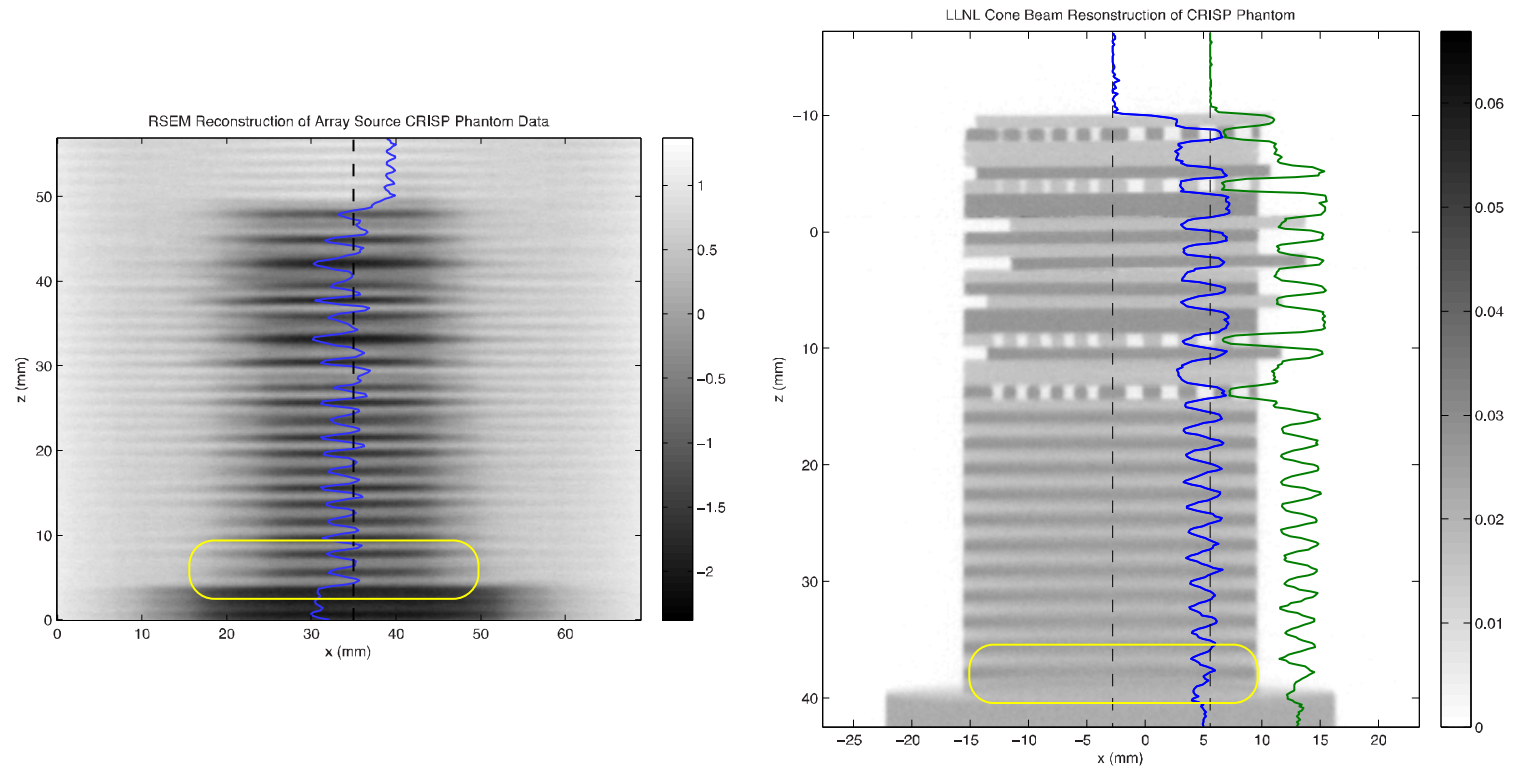

Figure 3: A reconstruction comparison of slices through the array source data (left) and a single source cone beam data (right). The array source data lacked sufficient photon count to achieve the contrast and resolution of the single source data, however cone beam artifacts are reduced in the array source data, as indicated by the yellow contours. 


\section{References:}

[1] S. K. Lehman, A. M. K. Foudray, "The Contrast and Resolution Interleaved Stacked Plate (CRISP) Phantom”, LLNL-TR-489551, June 2011 